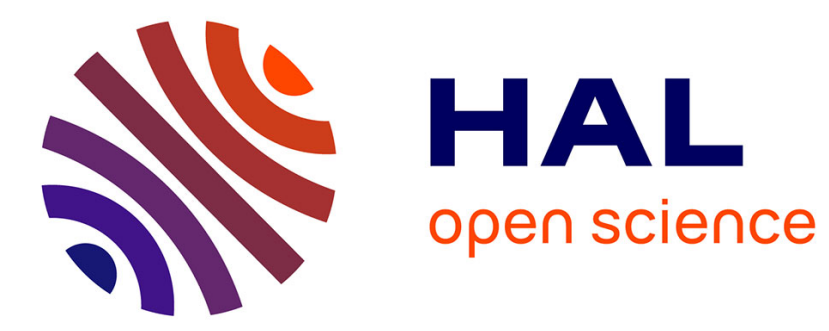

\title{
Moving the Boundaries of Granulopoiesis Modelling
}

Samuel Bernard

\section{To cite this version:}

Samuel Bernard. Moving the Boundaries of Granulopoiesis Modelling. Bulletin of Mathematical Biology, 2016, 78 (12), pp.2358 - 2363. 10.1007/s11538-016-0215-8 . hal-01391393

\section{HAL Id: hal-01391393 https://hal.inria.fr/hal-01391393}

Submitted on 15 Nov 2016

HAL is a multi-disciplinary open access archive for the deposit and dissemination of scientific research documents, whether they are published or not. The documents may come from teaching and research institutions in France or abroad, or from public or private research centers.
L'archive ouverte pluridisciplinaire HAL, est destinée au dépôt et à la diffusion de documents scientifiques de niveau recherche, publiés ou non, émanant des établissements d'enseignement et de recherche français ou étrangers, des laboratoires publics ou privés. 


\title{
Moving the boundaries of granulopoieis modelling
}

\author{
Samuel Bernard \\ Institut Camille Jordan/CNRS UMR5208i, Université de Lyon, Villeurbanne, France \\ bernard@math. univ-lyon1.fr
}

October 26, 2016

This is an author manuscript of the publication: Bernard, S. Bull Math Biol (2016) 78: 2358. doi:10.1007/s11538-016-0215-8

\section{Introduction}

The human blood cell production system usually remains extremely robust, in terms of cell number or function, with little signs of decline in old age. To achieve robustness, circulating blood cells rely on a formidable production machinery, the hematopoietic system, located in the bone marrow. All circulating blood cellsred blood cells, white blood cells and platelets - are renewed on a daily basis. The hematopoietic system produces an estimated $10^{12}$ cells per day. This is a significant fraction of the $3.7 \times 10^{13}$ cells in an adult $[5,1]$.

Robustness is partly due to the short time scales at which cell populations are able to return to equilibrium, combined with large cell numbers and renewal rates. White blood cells (WBCs), among which neutrophils are most prevalent, are the body's first line, innate immune system. Upon infection, WBCs are mobilized from the bone marrow, to increase their number in circulation and fight off pathogen within hours [3].

The 26 billion circulating neutrophils in human have a mean residence time of only $11 \mathrm{~h}$ in the blood [2]. After their release from the bone marrow, they quickly disappear in the peripheral tissues and are destroyed in the spleen, liver and bone marrow [3]. In addition to the high renewal rate of circulating blood cells, a large number of mature neutrophils, ten times or more the circulating number, is kept in a bone marrow reserve, ready for entering circulation. 
This high renewal rate and mobilization capability, however, come at a cost. The blood system is an easy target for chemotherapeutic drugs, whose main way of acting is by killing proliferating cells. White blood cells and end especially neutrophils, with their fast turnover, are particularly vulnerable to chemotherapy. Chemotherapy can induce neutropenia - a state of low absolute neutrophil count (ANC) - in cancer patients, which puts them at risk of infection.

Homeostatic regulation of white blood cells is mainly controlled by the cytokine Granulocye-Colony Stimulating Factor (G-CSF). G-CSF promotes survival of white blood cell precursors and their differentiation into mature cells. The identification of this protein in the 1980's [7], and the subsequent development of human recombinant forms of G-CSF paved the way to the treatment of chemotherapyinduced neutropenia. G-CSF therapy as also been successful at treating congenital and other forms of neutropenia.

Today, G-CSF is used as an adjuvant in several anti-cancer treatment protocols. The aim of the adjuvant therapy is to minimize the length of the neutropenic episodes. However, exogenous G-CSF administration interferes with white blood cell production regulation. What should be a straightforward effect-administer G-CSF to cause the ANC to increase - turns to be more complicated than that. For instance, it was observed that early timing of G-CSF administration could lead to prolonged neutropenic phase [8]. Thus, in order to take advantage of the full potential of G-CSF, a detailed understanding of the physiological interaction between neutrophils and exogenous G-CSF is necessary. In this issue of the Bulletin, Craig and colleagues present a physiological model of neutrophil production that includes a detailed modelling of the kinetics of G-CSF.

Mathematical modelling of white blood cell production has a long history [6]. The fundamental structure of all blood cell production models is the delayed negative feedback loop. The negative feedback loop is the key to a robust homeostasis; when cell number drops, blood cell production is increased, and cell number quickly returns to equilibrium. There is an intrinsic delay in the action of the negative loop, due to the finite time required to produce the extra cells needed. Dysregulation of parts of the negative feedback loop can lead to oscillation in circulating blood cell number. An example where this occurs is cyclic neutropenia, a genetic condition in which the ANC is oscillating with a period of around three weeks in human, and two weeks in the grey collie. Because the match is so good between clinical data and model predictions, the feeling is that the dynamics of the hematopoietic system dynamics is well characterised. This basic view of the negative feedback loop is too simple, however, if one is interested in short term perturbations of the hematopoietic system, following rounds of chemotherapy, for instance.

Negative feedback loop-based models of neutrophil production do not incorporate an explicit action of the G-CSF, and most do not include a mature neutrophil 
pool in the bone marrow. In their model, Craig and colleague have included a physiological pharmacokinetics/pharmacodynamics (PK/PD) model of G-CSF, in which they consider the effect of having a pool of bone marrow neutrophils on G-CSF kinetics, and on the recovery after chemotherapy-induced neutropenia.

It is important to model G-CSF explicitely because exogenous administration of G-CSF breaks the negative feedback loop: it is possible for the neutrophil count and the G-CSF concentration to be high at the same time. This mismatch lead earlier models to underestimate the contribution of neutrophil-mediated G-CSF clearance.

G-CSF is cleared by two distinct mechanisms, a linear renal clearance and G-CSF receptors on neutrophils. Bound G-CSF is internalized and subsequently degraded. After G-CSF administration, rapid bone-marrow neutrophil mobilisation and disappearance leads to a drop in neutrophil-mediated clearance. Craig and colleagues show convincing evindence that PK models neglecting the bone marrow neutrophil reserve overestimate renal clearance and taht neutrophil internalisation is the main clearance mechanism.

They present a model with five delay differential equations (DDEs), three for the neutrophil dynamics (stem cell number, bone marrow and circulating neutrophil count) and two for G-CSF (unbound and bound G-CSF). Mature bone marrow neutrophil $\left(N_{R}\right)$ include a state-dependent time delay $\tau_{N_{M(t)}}$, which satisfies a DDE itself. The time delay accounts for variable neutrophil maturations speeds. When building a DDE model with varying delays, it is tempting to take the equations with constant delays and just "add" the time or state-dependence to the delay terms. This is a mistake, though. The new equations with varying delays do not represent the intended model, and can lose desirable properties such positivity of solutions.

To see the difficulty with including varying delays, we consider an abstract regulation model, where the cell population of interest is a pool of mature cells. We assume that mature cells are produced at a positive rate $\beta$ dependending smoothly on the total number of cells $x$, and representing the rate of new cells produced after a constant maturation delay $\tau$.

A delay differential equation for $x(t)$ could be written as

$$
\frac{d x(t)}{d t}=\beta(x(t-\tau))-\gamma x(t),
$$

where $\gamma$ is a death rate. To take into account a time varying delay, $\tau \equiv \tau(t)$, it is tempting to modify the equation directly and write

$$
\frac{d x(t)}{d t}=\beta(x(t-\tau(t)))-\gamma x(t),
$$


but this formulation is incorrect. Indeed, if $\tau(t)=t$, no maturing cell can ever reach maturity and be released in the mature cell pool, even though the production rate $\beta(x(t-t))=\beta(x(0))$ is non zero.

In order to define an equation satisifying the modelling assumption that maturing cells spend a varying amount of time in maturation, it is necessary to look at the underlying transport equation that gives rise to the delay. The simplest transport equation that gives rise to a varying delay is

$$
\partial_{t} n(t, a)+v(t) \partial_{a} n(t, a)=0
$$

where $v(t)$ is a time varying maturation velocity and $a$ is the maturity level. If cells are released into the mature pool when they reach a maturity level $a=a_{M}$, then the time required for cell to become mature is defined implicitly by the relationship

$$
\tau(t)=\int_{t-\tau(t)}^{t} v(s) d s .
$$

From a maturation time viewpoint, equation (3) is a transport equation with a moving boundary. Differentiating equation (4) with respect to time yields

$$
1-\frac{d \tau(t)}{d t}=\frac{v(t)}{v(t-\tau(t))}
$$

New mature cells are produced at a rate $v(t) n\left(t, a_{M}\right)$, which is the flux of cell going trought the boundary $a=a_{M}$. The analogy of the conveyor belt introduced in [4] is useful to represent the effect of a varying speed and the boundaries (Fig 1A). It is convenient to assume $v(t) \geq 0$, to avoid cells from re-entering the maturation compartment at $a=a_{M}$ or leaving the compartment at $a=0$. In the same way, the flux of cells entering maturation is $v(t) n(t, 0)$. If we pose that the flux of cell entering maturation is $\beta(x(t)$, then we have the boundary condition

$$
v(t) n(t, 0)=\beta(x(t))
$$

Using the characteristics, we have that $n\left(t, a_{M}\right)=n(t-\tau(t), 0)$, and it follows that

$$
v(t) n\left(t, a_{M}\right)=v(t) n(t-\tau(t), 0)=\frac{v(t)}{v(t-\tau(t))} \beta(x(t-\tau(t))) .
$$

Therefore the correct form for the varying time delay equation is

$$
\frac{d x(t)}{d t}=\frac{v(t)}{v(t-\tau(t))} \beta(x(t-\tau(t)))-\gamma x(t),
$$

together with equation (5). If the form for $\tau(t)$ is known, the time varying delay equation is

$$
\frac{d x(t)}{d t}=\left(1-\frac{d \tau(t)}{d t}\right) \beta(x(t-\tau(t)))-\gamma x(t) .
$$


A

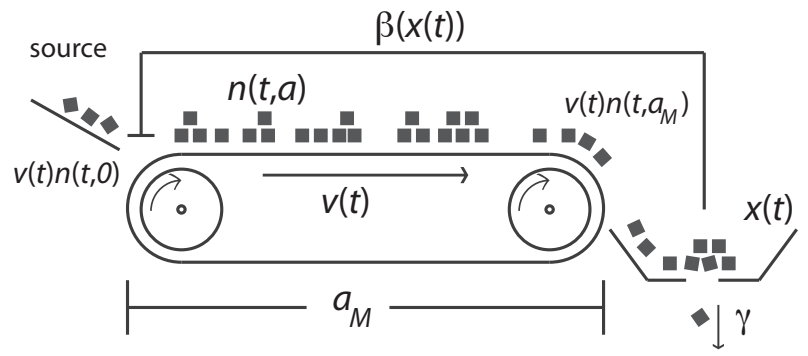

$\mathrm{B}$

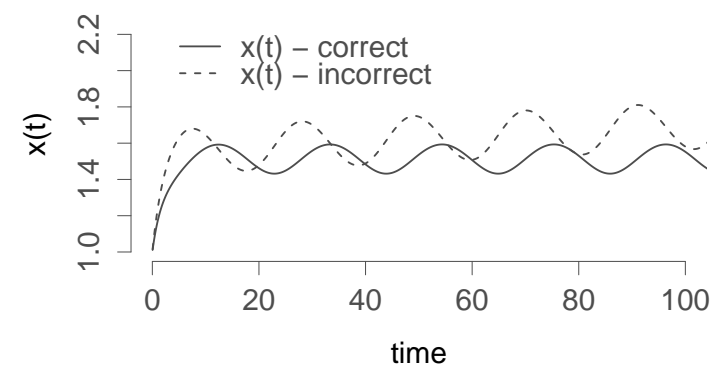

Figure 1: (A) The conveyor belt analogy [4]. Maturing cells are transported at speed $v(t)$. The production rate of mature cells is $v(t) n\left(t, a_{M}\right)$, while new cell enter the maturation compartment at a rate $\beta(x(t))=v(t) n(t, 0)$. (B) Simulations of equations (9) (solid) and (2) (dashed) with initial history $x(\theta)=1$ for $\theta \in\left[-\tau_{0}, 0\right], \beta(x)=k_{0} k^{2} /\left(k^{2}+x^{2}\right), \tau(t)=\tau_{0}+A \sin (2 \pi t / T)$, $\gamma=0.2, k_{0}=1.0, k=1.0, \tau_{0}=10.0, A=0.5, T=21$. 
Both descriptions valid representations. Equations $(5,8)$ uses a varying maturation velocity, while equation (9) uses a moving boundary for the length of the maturation phase. Equation (5) and non-negativity of $v(t)$ imply that $d \tau(t) / d t$ should never exceed 1. Equation (2) creates cells out of nowhere, leading to slow build up in cell number even though cell number should stabilise (Fig 1B). Craig and colleagues took care of keeping the books straight, making sure that all equations really represent the modelling assumptions.

In total, the full model possesses 32 kinetic parameters, and auxiliary equations for $\tau_{N}, \tau_{N_{M}}, A_{N}, A_{Q}$ due to non-constant delays, and additional equations for $\mathrm{PK} / \mathrm{PD}$ of the chemotherapy. Most of these parameters could be estimated from the literature, based on a combination of modelling and experimental measurements. The remaining parameters were estimated in a stepwise manner; first by characterising parameter constraints at homeostasis, then the G-CSF pharmacokinetics parameters and finally the neutrophil dynamics after IV or subcutaneous G-CSF administration.

Strong feedback mechanisms tend to become unstable, both dynamically and in terms of parameter sensitivity. To avoid that, Craig and colleagues used a combination of nonlinear least-square fitting together with objective functions that guaranteed robust fits. This way, Craig and colleagues could characterize not only physiological neutrophil dynamics, but also the out-of-equilibrium dynamics following G-CSF administration. A limitation with parameter estimation is the lack of data about stem cells. They used the obervation that G-CSF administration speeds up neutrophil precursor transit times to recover some of the stem cells kinetics.

The last parameter estimation step was made on individual patient chemotherapy. Craig and colleagues used neutrophil dynamics data during chemotherapy to estimate PK parameters. This way, they obtain a full PK/PD model of neutrophil dynamics during chemotherapy, with a detailed model for G-CSF administration.

To check the validity of the model, Craig and colleague simulated the CHOP14 protocol, which includes combined chemotherapy and G-CSF administration. They compared the simulation of six cycles of 14-day chemotherapy treatment with published CHOP14 data. Simulation predictions were very close to the published data, although no parameters were adjusted. These simulations show how the administration of G-CSF depletes the bone marrow neutrophil pool, and prevent circulating neutrophil counts from recovering. These results suggest that delaying G-CSF administration during chemotherapy would help minimise the severity of the neutropenia episodes.

This new model for granulopoiesis under exogenous G-CSF treatment illustrate the need for a realistic description of the physiology when trying to generate clin- 
ically relevant predictions. Including time delays into differential equation models has often been made too hastily, leading to modelling errors and raising doubts about the relevance of such models. This new paper shows how rigorous derivation of state-dependent time delays can move the boundaries of granulopoiesis modelling.

\section{References}

[1] Bianconi, E., Piovesan, A., Facchin, F., Beraudi, A., Casadei, R., Frabetti, F., Vitale, L., Pelleri, M.C., Tassani, S., Piva, F., et al.: An estimation of the number of cells in the human body. Annals of human biology 40(6), 463-471 (2013)

[2] Dancey, J., Deubelbeiss, K.A., Harker, L.A., Finch, C.A.: Neutrophil kinetics in man. Journal of Clinical Investigation 58(3), 705 (1976)

[3] Furze, R.C., Rankin, S.M.: Neutrophil mobilization and clearance in the bone marrow. Immunology 125(3), 281-288 (2008)

[4] Mahaffy, J.M., Bélair, J., Mackey, M.C.: Hematopoietic model with moving boundary condition and state dependent delay: applications in erythropoiesis. Journal of theoretical biology 190(2), 135-146 (1998)

[5] Ogawa, M.: Differentiation and proliferation of hematopoietic stem cells. Blood 81(11), 2844-2853 (1993)

[6] Pujo-Menjouet, L.: Blood cell dynamics: Half of a century of modelling. Mathematical Modelling of Natural Phenomena 11(1), 92-115 (2016)

[7] Welte, K., Gabrilove, J., Bronchud, M., Platzer, E., Morstyn, G.: Filgrastim (r-methug-csf): the first 10 years. Blood 88(6), 1907-1929 (1996)

[8] Zwick, C., Hartmann, F., Zeynalova, S., Pschel, V., Nickenig, C., Reiser, M., Lengfelder, E., Peter, N., Schlimok, G., Schubert, J., Schmitz, N., Loeffler, M., Pfreundschuh, M., for the German High-Grade Non-Hodgkin Lymphoma Study Group: Randomized comparison of pegfilgrastim day 4 versus day 2 for the prevention of chemotherapy-induced leukocytopenia. Annals of Oncology 22(8), 1872-1877 (2011). DOI 10.1093/annonc/mdq674. URL http://annonc.oxfordjournals.org/content/22/8/1872.abstract 